NBER WORKING PAPER SERIES

\title{
THE IMPACT OF DOMESTIC TRAVEL BANS ON COVID-19 IS NONLINEAR IN THEIR DURATION
}

\author{
Fiona Burlig \\ Anant Sudarshan \\ Garrison Schlauch \\ Working Paper 28699 \\ http://www.nber.org/papers/w28699 \\ NATIONAL BUREAU OF ECONOMIC RESEARCH \\ 1050 Massachusetts Avenue \\ Cambridge, MA 02138 \\ April 2021
}

Burlig and Sudarshan contributed equally to this work. We thank Susanna Berkouwer, Eyal Frank, Amir Jina, Erin Kelley, and Gregory Lane for helpful comments, and the crowdsourcing teams at https://www.covid19india.org/and https://github.com/GoogleCloudPlatform/covid-19open-data for producing public information on COVID-19 outbreaks. All errors are our own. The views expressed herein are those of the authors and do not necessarily reflect the views of the National Bureau of Economic Research.

NBER working papers are circulated for discussion and comment purposes. They have not been peer-reviewed or been subject to the review by the NBER Board of Directors that accompanies official NBER publications.

(C) 2021 by Fiona Burlig, Anant Sudarshan, and Garrison Schlauch. All rights reserved. Short sections of text, not to exceed two paragraphs, may be quoted without explicit permission provided that full credit, including $\odot$ notice, is given to the source. 
The Impact of Domestic Travel Bans on COVID-19 is Nonlinear in Their Duration

Fiona Burlig, Anant Sudarshan, and Garrison Schlauch

NBER Working Paper No. 28699

April 2021

JEL No. I18,J60,O12

\section{ABSTRACT}

Domestic mobility restrictions to control the spread of COVID-19 are widespread in developing countries, and have trapped millions of migrant workers in hotspot cities. We show that bans can increase cumulative infections relative to a counterfactual sans restrictions. A SEIR model shows bans' impacts are nonlinear in duration. We empirically test this hypothesis using a natural experiment in India as well as data from China, Indonesia, the Philippines, South Africa, and Kenya. Although very short and long restrictions limit the spread of disease, moderately lengthy restrictions substantially increase infections. This underscores the importance of considering duration in mobility-restricting policy decisions in developing countries.

Fiona Burlig

Harris School of Public Policy

University of Chicago

1307 East 60th Street

Chicago, IL 60637

and NBER

burlig@uchicago.edu

Anant Sudarshan

Department of Economics and

Energy Policy Institute at Chicago

University of Chicago

Saieh Hall

5757 S. University Avenue

Chicago, IL 60637

anants@uchicago.edu
Garrison Schlauch

Energy Policy Institute at the

University of Chicago (EPIC)

Saieh Hall for Economics

5757 S. University Ave

Chicago, IL 60637

USA

gschlauch@uchicago.edu

A data appendix is available at http://www.nber.org/data-appendix/w28699

A Code repository is available at https://github.com/fburlig/BSS_COVID_migration 
Governments around the world have deployed numerous policy instruments to control the spread of COVID-19 (Flaxman et al., 2020; Lai et al., 2020; Chinazzi et al., 2020; Hsiang et al., 2020; Ali et al., 2020; Kraemer et al., 2020). Some of these instruments, such as largescale lockdowns, are also associated with significant economic harm. These costs have been especially pronounced in developing countries, where economic slowdowns associated with COVID-19 policies combined with weak social safety nets were expected to push between 71-100 million people into extreme poverty in 2020 (World Bank Group, 2020a,b).

Domestic travel bans are a particularly severe and relatively common restriction. Their usage is motivated in part by simulation exercises that model them as effective methods for reducing the spread of disease while they are in effect (Li et al., 2020; Tang et al., 2020). However, they also impose substantial and inequitable economic costs (Bonaccorsi et al., 2020; Weill et al., 2020), making them difficult to sustain indefinitely. As a result, these policy instruments necessarily involve two decisions: (i) whether to restrict freedom of movement and (ii) for how long to do so.

Although travel restrictions have been used around the world, we focus on domestic travel bans implemented by developing countries. These nations are frequently characterized by the presence of large populations of migrant workers. A United Nations report that examines data from 70 countries and more than $70 \%$ of the global population found that more than 763 million people were living within their home country but outside their region of birth in 2005 (United Nations Population Division, 2013). For example, the National Bureau of Statistics in China reports approximately 277 million rural migrants in the country in 2015, with roughly 168 million having left villages for towns in a different hokou ( $\mathrm{Su}$, Tesfazion and Zhao, 2018). A 2017 Indian government report estimates that the country contains approximately 140 million domestic migrant laborers (Ministry of Finance, 2017), with more than 60 million of them moving across state boundaries. The type of rural-to-urban migration most affected by COVID-19 mobility restrictions is more common in developing countries than in the developed world (United Nations Population Division, 2013). The presence of a large population that may respond to economic shocks by moving has motivated many developing countries to utilize travel bans to prevent the spread of disease. 
In this paper, we estimate the impact of travel ban duration on the spread of COVID-19. We begin by simulating disease transmission using a standard SEIR model (Diekmann and Heesterbeek, 2000). Though highly stylized, this model mimics a real-world scenario facing many developing countries, in which migrants leaving an urban hotspot spread infections to a rural destination. The results from this modeling exercise generates our key hypothesis: that the impact of travel bans is nonlinear in their duration.

To test this empirically, we exploit quasi-experimental variation generated by a natural experiment in Mumbai, India - the country's financial capital and initial COVID-19 epicenter - which relaxed travel bans after varying durations. On March 25th, the country imposed a nationwide lockdown, maintaining a ban on domestic travel out of the city (Indian Ministry of Home Affairs, 2020). This lockdown caused immense suffering as the economy rapidly contracted and unemployment rose, especially among migrant workers, who do not have access to the social safety net in India. Under intense pressure (Supreme Court of India, 2020), the government allowed the first wave of migrants to return to homes outside Mumbai's state of Maharashtra on May 8th (Mehta, 2020) ('Phase 1'). 'Phase 2' migrants, returning to districts in the Mumbai Metropolitan Area, were allowed to leave on June 5th (Government of Maharashtra, Department of Revenue and Forest, Disaster Management, Relief and Rehabilitation, 2020a). Finally, 'Phase 3' migrants, departing to all other destinations, were able to leave on August 20th (News on AIR - News Services Division, All India Radio News, 2020). ${ }^{1}$ We use an event study design to estimate the impact of travel ban relaxation on COVID-19 cases in migrants' home locations, estimating separate treatment effects for each phase.

Finally, we use cross-country data to study whether our estimated impacts are likely to generalize to a broader context. For these comparisons, we examine travel bans in Indonesia, India, South Africa, the Philippines, China, and Kenya. Together, these countries comprise roughly $40 \%$ of the global population (Worldometers.info, 2021).

In both our SEIR simulations and our empirical results, we find that while relatively short and relatively long restrictions can successfully limit the spread of COVID-19, inter-

\footnotetext{
${ }^{1}$ We use the taxonomy of 'phases' for convenience, but these dates were not announced at once. For instance, the first release followed an Indian Supreme Court ruling forcing states to allow some movement in order to free trapped migrants.
} 
mediate length bans - once lifted - can significantly increase COVID-19 growth rates, cumulative infections, and deaths. The full effect of travel bans can therefore only be quantified after they are lifted. More broadly, our results underscore that quantifying the unintended consequences of COVID-19 restrictions, including both disease and economic costs, is critical for policy decisions.

\section{Simulating the impacts of domestic travel bans}

We begin by using a standard SEIR modeling framework (Diekmann and Heesterbeek, 2000) for COVID-19 as implemented in the R covoid package (Churches and Jorm, 2020; Fitzgerald, Hanly and Churches, 2020) to make theoretical predictions on the spread of COVID-19 once a domestic travel ban is lifted. We find that both short and long bans can lead to relatively few new infections, and that moderately lengthy bans can substantially increase total COVID-19 cases.

\section{A SEIR model}

We model a simplified setting that incorporates an urban hotspot (source) and a migrant origin location (sink). ${ }^{2}$ This scenario is typical of the developing countries in our empirical analysis and depicts a travel ban which prevents a large migrant population from leaving a city with a growing number of COVID-19 infections. At different points in time, a certain fraction of the source population is allowed to travel to the sink, initiating community transmission in the latter.

Through the lens of this model, two opposing forces constitute the first-order determinants of total infections at any point in time. On one hand, the longer a travel ban lasts, the less time community transmission exists in the rural sink. Ceteris paribus, this will decrease rural infections. On the other hand, the longer the restrictions remain, the longer migrants are contained within a hotspot where infection rates are rapidly increasing. Consequently, the probability that migrants are infected with COVID-19 rises over time until the city

\footnotetext{
${ }^{2}$ More specifically, our model simulates pandemic spread in a stylized setting consisting of an urban hotspot (source) with a rising number of infections and a rural destination (sink) with zero initial cases.
} 
achieves herd immunity, in turn increasing the rate at which they seed the rural sink with infections once the ban is lifted. This drives up cumulative cases at any future date.

Which of these two factors dominates is an empirical question whose answer depends on the reproduction rates $\left(R_{0}\right)$ in the source and sink, the number of returning migrants, the duration of travel restrictions, and the characteristics of the disease. We examine this tradeoff by parameterizing our SEIR model using values from the literature that correspond to Mumbai, India - a key location in our empirical analysis. This model compares total cases under travel bans of various durations to a counterfactual with no ban, in which migrants leave the source immediately.

In an SEIR model, people are modeled as transitioning between four conditions: 'Susceptible', 'Exposed', 'Infective', 'Recovered'. Four ordinary differential equations describe how the share of the population in each of these four conditions evolves over time:

$$
\begin{aligned}
\frac{d S(t)}{d t} & =-\theta I(t) \times \frac{s(t)}{N} \\
\frac{d E(t)}{d t} & =\theta I(t) \times \frac{S(t)}{N}-\omega E(t) \\
\frac{d I(t)}{d t} & =\omega E(t)-(\lambda) I(t) \\
\frac{d R(t)}{d t} & =(\lambda) I(t)
\end{aligned}
$$

The Susceptible fraction of the population $S$ is made up of those who could fall ill in the future due to virus exposure. The Exposed fraction $E$ are those who have already been infected but do not yet show symptoms. This category captures a latent period of infection. The Infected population $I$ is the share of people who have fallen ill and gone through the latent period. The Recovered population $R$ are those who have recovered from the infection with immunity or passed away. The key parameters in this set of equations are:

Infection rate $\theta$ is the infection rate, the number of people that are infected by a person in state $I$.

Latent period The latent period is the inverse of $\omega$ and represents the length of time in days spent in the exposed state $E$ before moving to state $I$. 
Infectious Period The infectious period ('recovery' period) is the inverse of $\lambda$ and represents the length of time over which the infection persists before running to completion. The infection may terminate in either a recovery or death. $\lambda$ averages over the times to both outcomes.

Reproduction number The reproduction number $R_{0}$ can be defined as the average number of secondary cases produced by one infected individual introduced into a population of susceptible individuals. This is an empirically important quantity because infections where $R_{0}$ is below 1 cannot spread exponentially through the population. In the context of the textbook model, $R_{0}=\theta S_{0} / \omega$. Note that $\theta$ and $R_{0}$ are not independent parameters as defined here.

In an SEIR framework, population size influences case growth. This implies that departing migrants will influence the propagation of cases in both the sink and source. This effect is second-order when the population size is large relative to the number of infections and departing migrants. Thus in this modelling exercise as well as our empirical analysis, we abstract away from the effect of departures on case growth in hotspots by defining excess cases based on rural infections.

We run the model with parameters chosen to approximate Mumbai's Phase 2 travel ban release. We fix some parameters directly from studies in the literature and calibrate others for consistency. ${ }^{3}$ The former category includes the hotspot and sink populations $\left(N_{h}, N_{s}\right)$ and the number of departing migrants.

The model runs described below require a reproduction number $R_{0}$ for both the source and sink. We calibrate the first of these such that the model predicts cumulative source infections $(R+I)$ that match the results of a large-scale serological study conducted by the Government of India, the Municipal Corporation of Mumbai, and the Tata Institute for Fundamental Research (Kolthur-Seetharam et al., 2020). To calibrate $R_{0}$ in this manner, we need to assume the number of seed infections at $t=0$ (March 25 in our simulations).

Due to limited testing at the time, no reliable case data exist. We assume 500 infections at time $t=0$. This assumption yields a calibrated $R_{0}$ value of 3.75 , which is comparable

\footnotetext{
${ }^{3}$ See Table A1 for parameter values and data sources.
} 
to estimates in the literature for the end of March in Delhi, India's capital and other early COVID-19 hotspot (Marimuthu et al., 2020b). This value seems reasonable - perhaps even conservative - in the context of people living in some of the most densely populated slums in the world and serves the purpose of producing an illustrative example. We assume $R_{0}$ for the rural sink is half the urban value, i.e. 1.875. This is similar to other estimates of $R_{0}$ for the state of Maharashtra at the end of March (Marimuthu et al., 2020a).

Having calibrated $R_{0}$ in this manner, we simulate cumulative urban and rural infections using a two step process. First, we simulate case growth in the urban source starting from March 25th $(t=0)$ until the travel ban release date at $t=T$, where $T$ ranges from $1-150$ days. Second, we simulate infections in both the source and sink. We set the initial exposed and infected seeds for the rural sink based on the probability of a departing migrant being in these conditions. That is, the number of infections entering the sink is $N_{m i g} \times(I(T) / N)$, where $N$ is the source population, $I(T)$ is the infected population at departure time $T$, and $N_{m i g}$ is the the departing population. The starting values of $R$ and $E$ for the rural sink can be similarly estimated. For each value of travel ban duration $T$, we predict cumulative infections $(I+R)$ in the rural sink up to September 30th, 189 days from the zero date of March 25th.

\section{B Simulation results}

Figure 1 plots excess total cases in the sink, normalized by the migrant population, against travel ban duration. Blue dots denote excess cases per 1,000 migrants for hypothetical short, intermediate, and long bans. For short travel bans, the number of infections in the sink is relatively low, but the period of time over community transmission occurs is relatively long. For long bans, there is less time for community transmission in the sink, and infections in the source begin to fall as as the population achieves herd immunity. For intermediate bans, net cases in the rural sink can significantly exceed those in the short or long ban counterfactuals. The definition of what would qualify as an 'intermediate' ban is depends on the specific context, but in this setting under reasonable parameter values, bans of intermediate duration are nevertheless predicted to be much worse than no restriction at all. 


\section{Data}

We briefly summarize our data sources and travel ban information here. More details including data cleaning steps are provided in our online Appendix. ${ }^{4}$

We collect COVID-19 epidemiological data for India, Indonesia, South Africa, Kenya, the Philippines, and China from a variety of national data sources collated by open-source organizations. We define COVID-19 hotspots as urban areas with significantly more cases than other regions in the same country in the period leading up to the travel ban release. Figure 2, Panel A reports case positivity rate in Mumbai, India's hotspot. Figure 3, Panel A reports hotspot cases per 1,000 people globally, as we do not observe testing rates outside India.

Information on travel ban dates for different countries was collected from news reports, government orders, and official press releases. ${ }^{5}$ All countries had nation-wide bans (which included travel restrictions to and from hotspots), except for China and Kenya, which banned travel to/from Wuhan, and Nairobi and Mombasa, respectively.

Migration data come from national government statistical agencies' censuses, enumerated in 2009 in Kenya, 2010 in Indonesia, China, and the Philippines, and 2011 in South Africa and India. Population data are obtained from WorldPop's constrained individual countries 2020 (100m resolution) datasets (WorldPop, 2020) and the 2011 Census of India (Registrar General of India, 2011).

The availability of epidemiological and migration data varies by country. We collect data at the most granular administrative unit available across these datasets. Data are at the province level in Indonesia, the district level in South Africa, the district level in India, the province level in China, the province level in the Philippines, and the district level in

\footnotetext{
${ }^{4}$ For a full list of data sources and travel ban information, see Tables A2 and A3.

${ }^{5}$ For both the country-level analysis and the Mumbai natural experiment, we use a \pm 30 day event window centered around travel ban release dates. Since these dates vary, the period of analysis for each country does as well. We use COVID-19 data from April 7th to June 6th in Indonesia, April 1st to May 31st in South Africa, April 8th to June 7th in India, March 9th to May 8th in China, April 30th to June 29th in the Philippines, and June 7th to August 6th in Kenya. The Mumbai travel ban involves three phases with event windows spanning from April 8th to June 7th, May 6th to July 5th, and July 21st to September 19th, respectively.
} 
Kenya. ${ }^{6}$ We merge the epidemiological, migration, and travel ban data at the administrative unit level for each country.

\section{Evidence from the Mumbai natural experiment}

The SEIR model describes a theoretical mechanism of disease transmission that is general but is nevertheless heavily simplified. Its main virtue is clarity. Parameters governing the spread of disease such as the reproduction rate are difficult to precisely quantify, particularly in an ongoing pandemic. Disease propagation in a real-world environment is characterized by a significant amount of spatial and temporal variation. We therefore require empirical evidence in order to draw rigorous conclusions about the impact of travel bans in practice.

Because many factors impact the spread of COVID-19, it is difficult to make causal statements about the relationship between travel ban durations and eventual cases. We overcome these challenges by leveraging a unique natural experiment in the city of Mumbai, India. India's travel ban policies allow us to compare the causal effect of bans of varying durations on infections in migrants' home regions, holding constant the urban hotspot.

This setting is substantively important. Mumbai is India's largest city, financial capital, and initial COVID-19 epicenter. India is home to over a sixth of the global population, imposed some of the world's strictest travel bans (Hale et al., 2020) early in the pandemic. Its 10 million recorded COVID-19 cases by the end of 2020 were second globally only to the United States (Dong, Du and Gardner, 2020).

COVID-19 and migrant workers Mumbai is one of India's top migrant destinations. According to the 2011 Census of India, approximately $43 \%$ of Mumbai's population originates from another state or district (Registrar General of India, 2011). Many domestic migrants in India are employed in the informal sector and have limited access to the social safety net, low engagement with formal banking, and transient addresses (Deshingkar and Akter, 2009; Mazumdar, Neetha and Agnihotri, 2013; De Haan, 2011). This made them particularly vulnerable to the economic consequences of India's COVID-19 lockdown (Sengupta and

\footnotetext{
${ }^{6}$ See our online Appendix for more details.
} 
Jha, 2020). Millions of migrant workers lost their jobs and tried to return home from Mumbai, often walking long distances with little food (Abi-Habib and Yasir, 2020; Bose, 2020; Suryawanshi, 2020a,c,b). This setting epitomizes the difficult trade-offs associated with imposing travel bans, highlighting the importance of understanding their impact on COVID-19 infections.

Travel restrictions On March 25th, 2020, India imposed a nationwide economic and mobility lockdown (Indian Ministry of Home Affairs, 2020). At this time, Mumbai was recording more new infections than any other Indian urban center. Social distancing interventions notwithstanding, by April 26th, the city had recorded more than 5,400 COVID-19 cases, by far the highest in the country at the time (COVID-19 India Org Data Operations Group, 2020).

For fear that millions of urban migrants would return home and export infections across rural India, the government maintained a ban on domestic travel out of the city, including shuttering airports, trains, and buses (Government of Maharashtra, Department of Revenue and Forest, Disaster Management, Relief and Rehabilitation, 2020b; Express Web Desk, 2020; Ministry of Home Affairs, Government of India, 2020). As the economy rapidly contracted (Press Information Bureau, Ministry of Statistics \& Programme Implementation, Government of India, 2020) and unemployment rates rose sharply (Centre for Monitoring Indian Economy, 2020), migrants began experiencing increasingly dire economic conditions (Abi-Habib and Yasir, 2020). Under intense pressure (Supreme Court of India, 2020), the government eventually lifted travel restrictions in three phases. Phase 1 migrants, returning to homes outside of the state of Maharashtra, were permitted to leave Mumbai as early as May 8th (Mehta, 2020). Phase 2 migrants, leaving for districts within the Mumbai Metropolitan Region, were able to depart on June 5th (Government of Maharashtra, Department of Revenue and Forest, Disaster Management, Relief and Rehabilitation, 2020a). Finally, Phase 3 migrants, going to all remaining destinations, were able to leave on August 20th (News on AIR - News Services Division, All India Radio News, 2020).

The Mumbai travel bans yield a natural experiment with a common infection source and three distinct travel resumptions. We use a panel fixed effects 'event study' design to 
study this policy. We describe the statistical analysis in detail below and our data sources in Table A2.

\section{A Empirical strategy}

We estimate the impact of domestic travel ban releases on COVID-19 cases for the three Mumbai travel bans and bans in other countries using a panel fixed effects ordinary least squares regression (Wooldridge, 2010; Angrist and Pischke, 2008). Formally, for each travel ban release, we estimate:

$$
I_{d t}=\sum_{s=-30}^{30} \beta^{s} 1[\text { days to release }=s]_{d t} \times \text { migrants }_{d}+\alpha_{d}+\delta_{t}+\varepsilon_{d t}
$$

where $I_{d t}$ is the number of new COVID-19 cases on date $t$ in administrative unit $d$, migrants $_{d}$ is the (static) number of migrants from administrative unit $d$ residing in the hotspot(s) as enumerated by national censuses, and 1 [days to release $=s]_{d t}$ is an indicator variable equal to 1 if date $t$ is $s$ days after the date of release to administrative unit $d$ and 0 otherwise. The latter indicator is calculated for every administrative unit $d$ for dates spanning 30 days before and after the release date $(s=-30$ to $s=30)$. As is evident from these definitions, $t$ refers to calendar time and $s$ refers to 'event time', which is defined relative to the release date. Negative values of $s$ imply dates that precede the travel ban release. We drop hotspots in order to, consistent with our SEIR model, isolate the effects of travel ban releases on infections in migrants' home regions.

This model non-parametrically controls for time-invariant and unobserved factors influencing new COVID-19 cases in each administrative unit, such as demographics, income, and healthcare quality, through the use of a full set of unit fixed effects $\alpha_{d}$. It also nonparametrically controls for common factors influencing new COVID-19 cases over time, for example country-wide under-detection of COVID-19 cases, seasonality that may affect disease spread, and changes in disease containment measures, through date-of-sample fixed effects $\delta_{t}$. These fixed effects also absorb the un-interacted 1 [days to release $\left.=s\right]_{d t}$ and migrants $_{d}$ terms. $\varepsilon_{d t}$ is an error term, two-way clustered by administrative unit and date- 
of-sample to account for arbitrary within-unit correlation and spatial correlation across administrative units (Cameron and Miller, 2015; Bertrand, Duflo and Mullainathan, 2004).

In this specification, the regression coefficients of interest, $\beta^{s}$, reflect the impact of travel ban releases on COVID-19 cases per migrant for each day $s$ after release. In the absence of any trends before the event, we could use these coefficients to estimate excess cases per migrant in the 30 days after each release given by $\sum_{s=0}^{30} \hat{\beta}^{s}$. In practice, for a given model specification, the assumption that pre-trends are zero (flat) may not perfectly hold. In such a case, post-release $\hat{\beta}^{s}$ values cannot be added to obtain a measure of excess cases. For example, consider a situation where equation (1) returns a similarly increasing trend of $\hat{\beta}^{s}$ coefficients before and after the event. This pattern should be interpreted as evidence of a zero treatment effect. However, simply adding up post-period $\hat{\beta}^{s}$ coefficients would yield a very different conclusion.

To guard against this possibility, we first adjust the $\hat{\beta}^{s}$ values before using them to estimate excess cases. Intuitively, what is required is a rotation of the estimated $\hat{\beta}^{s}$ coefficients about the omitted time dummy (we use $t=-1$ following standard practice), until a linear fit to $\hat{\beta}^{s}$ coefficients in the pre-period lies along the $\mathrm{x}$-axis. This rotation will also modify post-period coefficients, correctly removing the effect of any residual pre-trend that may have remained after estimating equation (1).

We implement this adjustment in a fairly straightforward manner. First, we estimate the slope of the pre-release coefficients by regressing

$$
\hat{\beta}^{s}=\theta s+\eta_{s}
$$

for $s<0$, where $s$ is in event time and $\eta_{s}$ is an error term. Next, we calculate adjusted estimates

$$
\tilde{\beta}^{s}=\hat{\beta}^{s}-(\hat{\theta}(s+1)) .
$$

We use $s+1$ (rather than $s$ alone) because the omitted dummy in our regressions is set at $t=-1$, and this adjustment correctly preserves this point while 'rotating' the original $\hat{\beta}^{s}$ coefficients about it. When the estimated $\hat{\theta}$ is zero, the adjusted $\tilde{\beta}^{s}$ coefficients equal 
the unadjusted $\hat{\beta}^{s}$ coefficients. If a pre-trend exists, however, this adjustment is critical to eliminating bias.

In practice, this adjustment makes our estimates more conservative. Without this adjustment, we slightly overestimate excess cases per migrant following the Phase 1 and Phase 2 travel ban releases in India, and substantially overestimate effects for Phase 3 (Figure A1). Our cross-country results are similar with or without this adjustment (Figure A2).

Taking the adjusted $\tilde{\beta}^{s}$ coefficients, we calculate excess COVID-19 cases per migrant in the 30 days after each travel ban release as

$$
\text { excess cases per migrant }=\sum_{s=0}^{30} \tilde{\beta}^{s}
$$

taking care to appropriately compute the variance for a sum of random variables. This quantity is a useful indicator for the growth rate of new infections that are generated in migrants' home administrative units following travel ban releases.

To compute excess case estimates comparable to those generated by the SEIR model, we re-estimate equation (1) with a fixed endpoint:

$$
\begin{gathered}
I_{d t}=\sum_{s=-30}^{T} \beta^{s} 1[\text { days to release }=s]_{d t} \times \text { migrants }_{d} \\
+\alpha_{d}+\delta_{t}+\varepsilon_{d t} .
\end{gathered}
$$

Because the Phase 3 release occurs significantly later than the Phase 1 and Phase 2 releases, we examine the latter two and fix the endpoint to June 30th for each $(T=53$ for Phase 1 and $T=25$ for Phase 2). We then compute excess cases per migrant in the $T$ days after each release as described by equation (4), with the coefficients summing to $s=T$ instead of $s=30$ :

$$
\text { excess cases per migrant }=\sum_{s=0}^{T} \tilde{\beta}^{s} \text {. }
$$

To interpret our effects as causal, we require a conditional independence assumption:

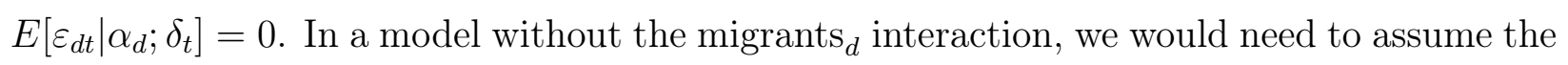


timing of travel ban releases was unrelated to home administrative unit cases and common counterfactual trends. Because our model interacts the treatment timing dummy variables with the number of migrants from each home administrative unit $d$, we require a substantially weaker assumption: parallel counterfactual trends in migrant-scaled home administrative unit cases, conditional on our fixed effects.

A standard test of this assumption is to examine the trend in home administrative units prior to and following a sharp 'treatment' (travel ban lifting). In Figure 2, panel C, we plot $\tilde{\beta}^{s}$ event study coefficients for Mumbai's three ban releases. We see a sharp trend break for the Phase 1 and Phase 2 releases, consistent with this assumption. In line with our aggregated Phase 3 estimate not being statistically significantly different from zero, we do not see a sharp break for Phase 3 .

Furthermore, we find no evidence that travel ban releases impacted COVID-19 cases when we estimate our model without scaling home administrative units by the number of migrants (Figure A1). This is important evidence in favor of our identifying assumption: any confounder must differentially impact home administrative units in a manner coincident with the timing of ban releases, and also supports our hypothesis that migrants are the cause of increased COVID-19 cases following travel ban releases.

\section{B Results}

Figure 2, Panel A, shows the COVID-19 test positivity rate in Mumbai over time, a measure of infection intensity in the hotspot. As is clear, the positivity rate starts off low, rises rapidly, and eventually falls. We observe a similar pattern using raw case counts.

Figure 2, Panel B, plots the home districts of Mumbai migrants, using data from the 2011 Census of India. ${ }^{7}$ We find substantial variation across districts. Amidst the chaos of Mumbai's travel restrictions, obtaining official statistics on the number and destinations of people who fled the city is impossible. We therefore use the distribution of home districts of recorded migrants as a (time-invariant) proxy for the destinations and relative shares of migrants who left the city.

\footnotetext{
${ }^{7}$ See our online Appendix for more details.
} 
Figure 2, Panel C, shows results from the event study regression model we use to estimate the excess cases generated in migrants' home districts after each of Mumbai's travel ban releases. This approach uses the evolution of COVID-19 cases in each migrant home district from before a ban release to construct a counterfactual describing what would have happened had travel not been permitted. If lifting a ban leads to faster infection growth in migrants' home districts, this will cause a trend break shortly after the date movement is allowed. From top to bottom, we show results for Phases 1, 2, and 3. The x-axis is a normalized time-scale, running from 30 days before to 30 days after each travel ban release, where $t=0$ is the release date. The $\mathrm{y}$-axis is the number of excess cases in migrants' home districts, compared to a counterfactual without a ban release.

For all three phases, our estimate of excess cases hovers around zero until each ban release, consistent with our hypothesis that active travel bans temporarily limited disease transmission from Mumbai to sink districts. After the bans are relaxed, we find that cases rise somewhat in Phase 1 districts (relatively short ban), substantially in Phase 2 districts (intermediate ban), and negligibly in Phase 3 districts (relatively long ban). The area under each curve where $t \geq 0$ measures cumulative excess cases in the 30 days after each ban release.

Although Figure 2, Panel C, demonstrates that both short and intermediate length travel bans played a key role in increasing rural infections, the observed magnitudes are a function of both the number of returning migrants in each Phase and the probability that a given migrant carries the disease. In Figure 2, Panel D, we plot a normalized statistic: excess cases per 1,000 Census migrants within 30 days of ban release to districts in each phase.

We observe a clear parabolic relationship. On a per migrant basis, the intermediate length ban generated the largest effect, while short and long bans produced far fewer cases. This suggests that short (Phase 1) and long (Phase 3) bans, which ended when hotspot cases were relatively low (Figure 2, Panel A), led to low infection growth rates in migrants' home districts, while the intermediate length (Phase 2) ban caused rapid infection growth. We 
find similar, albeit noisier, results using daily COVID-19 deaths as the dependent variable rather than daily COVID-19 cases. $^{8}$

Finally, Figure 2, Panel E, compares excess COVID-19 cases per 1,000 Census migrants in destination districts following the short (Phase 1) and intermediate (Phase 2) ban, counting cumulative cases occurring through a fixed end date of June 30 th. $^{9}$ We compute this using coefficients from an extended version of the event study from Figure 2, Panel C, where we count excess cases up to 53 days after the Phase 1 release and 25 days after the Phase 2 release (equations 5 and 6 ). This accounts for both opposing forces in our SEIR model. Phase 1 migrants have more time to spread infections, while Phase 2 migrants are more likely to be infected before returning home. We find that Phase 2 districts experienced more new cases per Census migrant, even in a shorter 25 day period. In this empirical setting, the lengthier travel restriction created more total infections than the shorter ban.

\section{C Robustness}

Our estimates of per migrant excess cases, computed as described above, are robust to a variety of alternative specifications, including removing the date-of-sample fixed effects; replacing the date-of-sample fixed effects with a polynomial in sample date; adding a control

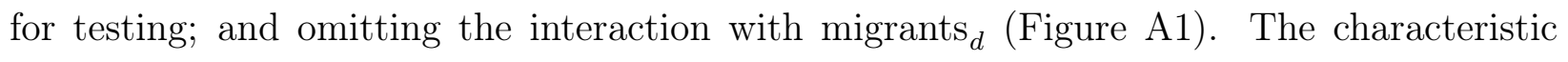
parabolic relationship between travel ban duration the COVID-19 cases per migrant in visible across specifications.

In Figure A4, we conduct a permutation inference test for the Mumbai travel ban release analysis. First, we reassign each district's COVID-19 case time series to a randomly-selected district without replacement. Then, we re-estimate our regression model (equation 1), adjust for linear trends in the pre-release coefficients (equations 2 and 3). Finally, we compute excess cases per-migrant by summing the post-release coefficients. We repeat this process 10,000 times and plot the resulting point estimates. This yields permutation inference $p$-values of $<0.0001$ for Phase 1, 0.0008 for Phase 2, and 0.4395 for Phase 3, suggesting that our estimates are not spurious.

\footnotetext{
${ }^{8}$ See Figure A3 and the online Appendix for details.

${ }^{9}$ This figure uses Indian data from April 8th to June 30th.
} 


\section{Cross-country comparisons}

We now investigate the relationship between the duration of domestic travel bans and the impact of migrants on COVID-19 cases around the developing world. We collected subnational data on travel bans and COVID-19 cases in Indonesia, India, South Africa, the Philippines, China, and Kenya. Together, these countries comprise roughly $40 \%$ of the global population (Worldometers.info, 2021).

We link the travel ban dates in each country with national statistics data on domestic migration and COVID-19 cases. ${ }^{10}$ For each country, we identify one or more hotspots, defined as locations with relatively high COVID-19 cases that restricted travel to other regions, ${ }^{11}$ and study the impact of the first national travel ban release.

In Figure 3, Panel A, we plot the number of hotspot COVID-19 cases per capita within one week of travel ban relaxation, along with a quadratic fit. As in Mumbai (Figure 2, Panel A), per-capita hotspot infections are relatively low at the time of short and long ban releases, and relatively high at the time of intermediate length releases.

Analogous to Figure 2, Panel D, in Figure 3, Panel B, we plot excess COVID-19 cases per 1,000 Census migrants, estimated using a similar country-specific event study design. Unlike Figure 2, we cannot rule out the influence of other factors that differ across countries. This caveat notwithstanding, we find a clear parabolic relationship across estimates, consistent with our theoretical predictions and our results from Mumbai.

First, we see no evidence that Indonesia's short travel ban significantly increased rural infections once migrants were allowed to return home. Second, the ending of intermediate length bans in South Africa and India produced substantial excess cases. In the Philippines, which had a slightly longer ban duration, we continue to find evidence that ban releases increased cases, but to a lesser extent than India or South Africa. We see little evidence that Kenya's long travel ban increased rural cases. In China, hotspot cases had fallen more quickly than in other countries, nearing zero at the time of ban release. We correspondingly find no evidence of new cases following the travel ban relaxation. These cross-country estimates

\footnotetext{
${ }^{10}$ See our online Appendix for details.

${ }^{11}$ Ibid.
} 
suggest that our Mumbai results, showing that the impact of travel bans on COVID-19 cases is nonlinear in their duration, generalize around the developing world.

Similarly to our results for the Mumbai natural experiment, our cross-country comparisons are robust to removing the date-of-sample fixed effects and replacing the date-of-sample fixed effects with a polynomial in the sample date (Figure A2). Due to data constraints, we are unable to control for subnational tests in countries other than India. However, countrywide trends in testing that vary over time are picked up by the date-of-sample fixed effects. Because our samples for other countries include a single ban release, removing the migrants ${ }_{d}$ interaction results in collinearity between the 1 [days to release $=s]_{d t}$ term and the date-ofsample fixed effects, so we omit this robustness check for the cross-country results.

\section{Discussion}

Because the costs of travel bans directly influence their feasible durations, decisions on whether to implement bans cannot be made independently from their likely economic impacts, which will vary by local context. This is true even if we measure the desirability of policy outcomes purely in terms of COVID-19 cases or deaths. The available evidence suggests that severe lockdowns can be extremely costly (Press Information Bureau, Ministry of Statistics \& Programme Implementation, Government of India, 2020; Centre for Monitoring Indian Economy, 2020; Ravindran and Shah, 2020; Abi-Habib and Yasir, 2020). Our results provide evidence that domestic travel bans can also produce unintended consequences with respect to disease transmission. Our analysis is not without limitation: the chaotic nature of mobility in developing countries in the wake of these releases makes it impossible to observe actual leaver counts. However, our robustness checks (Figures A1, A2, and A4) demonstrate that migrants are a key source of disease transmission after travel ban release, and our empirical estimates are consistent with the results from our theoretical model. Secondly, our cross-country analysis necessarily cannot cannot account for unobserved factors that may differ across countries. The fact that this analysis produces similar results to our Mumbai natural experiment, which holds constant the source of infection, gives us confidence in its validity. 
Taken together, our findings suggest that domestic travel bans can lead to an increase in COVID-19 cases if policymakers cannot commit to sufficiently long restrictions. The importance of accounting for duration when projecting the impacts of domestic travel bans also implies that empirical estimates based on data collected before travel restrictions were lifted may be misleading. Had the Mumbai restrictions been evaluated before migrants were allowed to leave, as in prior work which examined travel restrictions in other settings (Hsiang et al., 2020; Flaxman et al., 2020), one might have erroneously concluded that infections were reduced because community transmission in rural India would have been temporarily prevented.

That said, even bans that increases cumulative infections will delay the occurrence of new cases through community spread outside hotspots. If policymakers could use this time to significantly improve the availability of healthcare, the number of serious complications and deaths may fall even as total infections rise. Although this could serve as a legitimate justification for using travel bans, it is likely easier said than done in most developing countries, where rural public health services were often inadequate and overburdened well before the coronavirus pandemic. 


\section{References}

Abi-Habib, Maria, and Sameer Yasir. 2020. "India's coronavirus lockdown leaves vast numbers stranded and hungry." New York Times. https://www.nytimes.com/2020/03/29/ world/asia/coronavirus-india-migrants.html.

Ali, Sheikh Taslim, Lin Wang, Eric HY Lau, Xiao-Ke Xu, Zhanwei Du, Ye Wu, Gabriel M Leung, and Benjamin J Cowling. 2020. "Serial interval of SARS-CoV-2 was shortened over time by nonpharmaceutical interventions." Science, 369(6507): 11061109.

Angrist, Joshua D., and Jörn-Steffen Pischke. 2008. Mostly harmless econometrics: An empiricist's companion. Princeton University Press.

Bertrand, Marianne, Esther Duflo, and Sendhil Mullainathan. 2004. "How much should we trust differences-in-differences estimates?" Quarterly Journal of Economics, 119(1): 249-275.

Bonaccorsi, Giovanni, Francesco Pierri, Matteo Cinelli, Andrea Flori, Alessandro Galeazzi, Francesco Porcelli, Ana Lucia Schmidt, Carlo Michele Valensise, Antonio Scala, Walter Quattrociocchi, et al. 2020. "Economic and social consequences of human mobility restrictions under COVID-19." Proceedings of the National Academy of Sciences, 117(27): 15530-15535.

Bose, Mrityunjay. 2020. "Coronavirus lockdown: Nearly 17 lakh migrants left Maharashtra in one month." Deccan Herald. https://www.deccanherald.com/national/west/ coronavirus-lockdown-nearly-17-lakh-migrants-left-maharashtra-in-one-month-844388. html.

Cameron, A. Colin, and Douglas L. Miller. 2015. "A practitioner's guide to clusterrobust inference." Journal of Human Resources, 50(2): 317-372.

Centre for Monitoring Indian Economy. 2020. "Unemployment rate monthly time series (\%): Urban." https://unemploymentinindia.cmie.com/kommon/bin/sr.php?kall= wsttimeseries\&index_code $=050050000000 \&$ dtype $=$ urban.

Chinazzi, Matteo, Jessica T Davis, Marco Ajelli, Corrado Gioannini, Maria Litvinova, Stefano Merler, Ana Pastore y Piontti, Kunpeng Mu, Luca Rossi, Kaiyuan Sun, et al. 2020. "The effect of travel restrictions on the spread of the 2019 novel coronavirus (COVID-19) outbreak." Science, 368(6489): 395-400.

Churches, Timothy, and Louisa Jorm. 2020. "Flexible, freely available stochastic individual contact model for exploring COVID-19 intervention and control strategies: Development and simulation." JMIR Public Health and Surveillance, 3(6): e18965.

COVID-19 India Org Data Operations Group. 2020. "Dataset for tracking COVID-19 spread in India." https://api.covid19india.org/.

De Haan, Arjan. 2011. "Inclusive Growth?: Labour Migration and Poverty in India." The Indian Journal of Labour Economics, 54(3): 387-409.

Deshingkar, Priya, and Shaheen Akter. 2009. "Migration and human development in India." Human Development Report Office (HDRO), United Nations Development Programme (UNDP). HDRP-2009-13.

Diekmann, O, and JAP Heesterbeek. 2000. "Mathematical epidemiology of infectious diseases: Model building, analysis and interpretation." 
Dong, Ensheng, Hongru Du, and Lauren Gardner. 2020. "An interactive web-based dashboard to track COVID-19 in real time." The Lancet infectious diseases, 20(5): 533-534.

Express Web Desk. 2020. "Highlights: Maharashtra extends lockdown till August 31." The Indian Express. https://indianexpress.com/article/cities/mumbai/coronavirusmaharashtra-mumbai-pune- thane-live-latest-news-updates-today-covid-19-corona-cases$6524709 /$.

Fitzgerald, Oisin, Mark Hanly, and Timothy Churches. 2020. "covoid: COVID-19 open-source infection dynamics."

Flaxman, Seth, Swapnil Mishra, Axel Gandy, H Juliette T Unwin, Thomas A Mellan, Helen Coupland, Charles Whittaker, Harrison Zhu, Tresnia Berah, Jeffrey W Eaton, et al. 2020. "Estimating the effects of non-pharmaceutical interventions on COVID-19 in Europe." Nature, 584(7820): 257-261.

Government of Maharashtra, Department of Revenue and Forest, Disaster Management, Relief and Rehabilitation. 2020a. "Order No. DMU/2020/CR. 92/DisM-1."

Government of Maharashtra, Department of Revenue and Forest, Disaster Management, Relief and Rehabilitation. 2020b. "Order No. DMU/2020/CR. 92/DisM1." https://www.maharashtra.gov.in/Site/Upload/Government\%20Resolutions/Marathi/ 202005051315302819.pdf.

Hale, Thomas, Noam Angrist, Emily Cameron-Blake, Laura Hallas, Beatriz Kira, Saptarshi Majumdar, Anna Petherick, Toby Phillips, Helen Tatlow, and Samuel Webster. 2020. "Oxford COVID-19 Government Response Tracker, Blavatnik School of Government." https://raw.githubusercontent.com/OxCGRT/covid-policytracker/master/data/OxCGRT_latest.csv.

Hsiang, Solomon, Daniel Allen, Sébastien Annan-Phan, Kendon Bell, Ian Bolliger, Trinetta Chong, Hannah Druckenmiller, Luna Yue Huang, Andrew Hultgren, Emma Krasovich, et al. 2020. "The effect of large-scale anti-contagion policies on the COVID-19 pandemic." Nature, 584(7820): 262-267.

Indian Ministry of Home Affairs. 2020. "Order No.40-3/2020-DM-I (A)." https://www.mha.gov.in/sites/default/files/PR_Consolidated\%20Guideline\%20of\% 20MHA_28032020\%20\%281\%29_1.PDF.

Kolthur-Seetharam, Ullas, Daksha Shah, Jayanthi Shastri, Sandeep Juneja, Gagandeep Kang, Anup Malani, Manoj Mohanan, Gayatri Nair Lobo, Gajanan Velhal, and Mangla Gomare. 2020. "SARS-CoV2 serological survey in Mumbai by NITI-BMC-TIFR." NITI-Aayog, Municipal Corporation of Greater Mumbai and the Tata Institute of Fundamental Research.

Kraemer, Moritz UG, Chia-Hung Yang, Bernardo Gutierrez, Chieh-Hsi Wu, Brennan Klein, David M Pigott, Louis Du Plessis, Nuno R Faria, Ruoran Li, William P Hanage, et al. 2020. "The effect of human mobility and control measures on the COVID-19 epidemic in China." Science, 368(6490): 493-497.

Lai, Shengjie, Nick W Ruktanonchai, Liangcai Zhou, Olivia Prosper, Wei Luo, Jessica R Floyd, Amy Wesolowski, Mauricio Santillana, Chi Zhang, Xiangjun Du, et al. 2020. "Effect of non-pharmaceutical interventions to contain COVID-19 in China." Nature. 
Li, Ruiyun, Sen Pei, Bin Chen, Yimeng Song, Tao Zhang, Wan Yang, and Jeffrey Shaman. 2020. "Substantial undocumented infection facilitates the rapid dissemination of novel coronavirus (SARS-CoV-2)." Science, 368(6490): 489-493.

Marimuthu, S, Melvin Joy, B Malavika, Ambily Nadaraj, Edwin Sam Asirvatham, and L Jeyaseelan. 2020a. "Modelling of reproduction number for COVID-19 in India and high incidence states." Clinical Epidemiology and Global Health.

Marimuthu, S., Melvin Joy, B. Malavika, Ambily Nadaraj, Edwin Sam Sam, Asirvatham, and L. Jeyaseelan. 2020b. "Modelling of reproduction number for COVID19 in India and high incidence states." Clinical Epidemiology and Global Health. Preprint, https://www.sciencedirect.com/science/article/pii/S221339842030169X.

Mazumdar, Indrani, N Neetha, and Indu Agnihotri. 2013. "Migration and gender in India." Economic and Political Weekly, 54-64.

Mehta, Manthan K. 2020. "First train from Mumbai leaves with Uttar Pradesh migrants." The Times of India. https://timesofindia.indiatimes.com/city/mumbai/first-train-frommumbai-leaves-with-uttar-pradesh-migrants/articleshow/75632159.cms.

Ministry of Finance. 2017. Economic Survey 2016-17. Government of India.

Ministry of Home Affairs, Government of India. 2020. "Order No. 40-3/2020-DMI(A)." https://timesofindia.indiatimes.com/photo/75152283.cms.

News on AIR-News Services Division, All India Radio News. 2020. "Maharashtra govt permits resumption of inter-district bus transport service." https://newsonair.gov.in/News?title=Maharashtra-govt-permits-resumption-of-interdistrict-bus-transport-service\&id=397699.

Press Information Bureau, Ministry of Statistics \& Programme Implementation, Government of India. 2020. "Estimates of gross domestic product for the first quarter of April-June of 2020-21." Press Release, https://pib.gov.in/PressReleasePage.aspx?PRID= 1650021.

Ravindran, Saravana, and Manisha Shah. 2020. "Unintended consequences of lockdowns: Covid-19 and the shadow pandemic." National Bureau of Economic Research.

Registrar General of India. 2011. "2011 Census of India.” www.censusindia.gov.in/ 2011census/migration.html.

Sengupta, Sohini, and Manish K Jha. 2020. "Social policy, COVID-19 and impoverished migrants: Challenges and prospects in locked down India." The International Journal of Community and Social Development, 2(2): 152-172.

Supreme Court of India. 2020. "The problems and miseries of migrant labourers." https://main.sci.gov.in/supremecourt/2020/11706/11706_2020_34_1501_22499_ Order_09-Jun-2020.pdf.

Suryawanshi, Sudhir. 2020a. "COVID-19: As India begins to 'unlock', migrant workers flocking back to Mumbai for work." The New Indian Express. https://www.newindianexpress.com/thesundaystandard/2020/jul/05/covid-19-as-indiabegins-to-unlock-migrant-workers-flocking-back-to-mumbai-for-work-2165468.html. 
Suryawanshi, Sudhir. 2020b. "COVID-19 lockdown, reverse migration delays pre-monsoon agricultural work." The New Indian Express. https://www.newindianexpress.com/nation/ 2020/may/31/covid-19-lockdown-reverse-migration-delays-pre-monsoon-agriculturalwork-2150471.html.

Suryawanshi, Sudhir. 2020c. "Uddhav government turns blind eye to migrants leaving sans permission as exodus from Maharashtra continues." The New Indian Express. https://www.newindianexpress.com/nation/2020/may/16/uddhav-government-turnsblind-eye-to-migrants-leaving-sans-permission-as-exodus-from-maharashtra-continues2144224.html.

Su, Yaqin, Petros Tesfazion, and Zhong Zhao. 2018. "Where are the migrants from? Inter- vs. intra-provincial rural-urban migration in China." China Economic Review, 47: $142-155$.

Tang, Biao, Xia Wang, Qian Li, Nicola Luigi Bragazzi, Sanyi Tang, Yanni Xiao, and Jianhou Wu. 2020. "Estimation of the transmission risk of the 2019-nCoV and its implication for public health interventions." Journal of Clinical Medicine, 9(2): 462.

United Nations Population Division. 2013. "Cross-national comparisons of internal migration: An update on global patterns and trends." United Nations. Technical Paper No. $2013 / 1$.

Weill, Joakim A., Matthieu Stigler, Olivier Deschenes, and Michael R. Springborn. 2020. "Social distancing responses to COVID-19 emergency declarations strongly differentiated by income." Proceedings of the National Academy of Sciences, 117(33): 19658-19660.

Wooldridge, Jeffrey M. 2010. Econometric analysis of cross section and panel data. Vol. 1 of MIT Press Books, The MIT Press.

World Bank Group. 2020a. June 2020: Global economic prospects. The World Bank.

World Bank Group. 2020b. "Projected poverty impacts of COVID-19 (coronavirus)."

Worldometers.info. 2021. "Countries in the world by population." https://www. worldometers.info/world-population/population-by-country/.

WorldPop. 2020. "Constrained individual countries 2020 (100m resolution)." https://www. worldpop.org/geodata/listing?id=78. 


\section{Figures}

Figure 1: Predicted infections following travel ban releases are nonlinear in ban duration

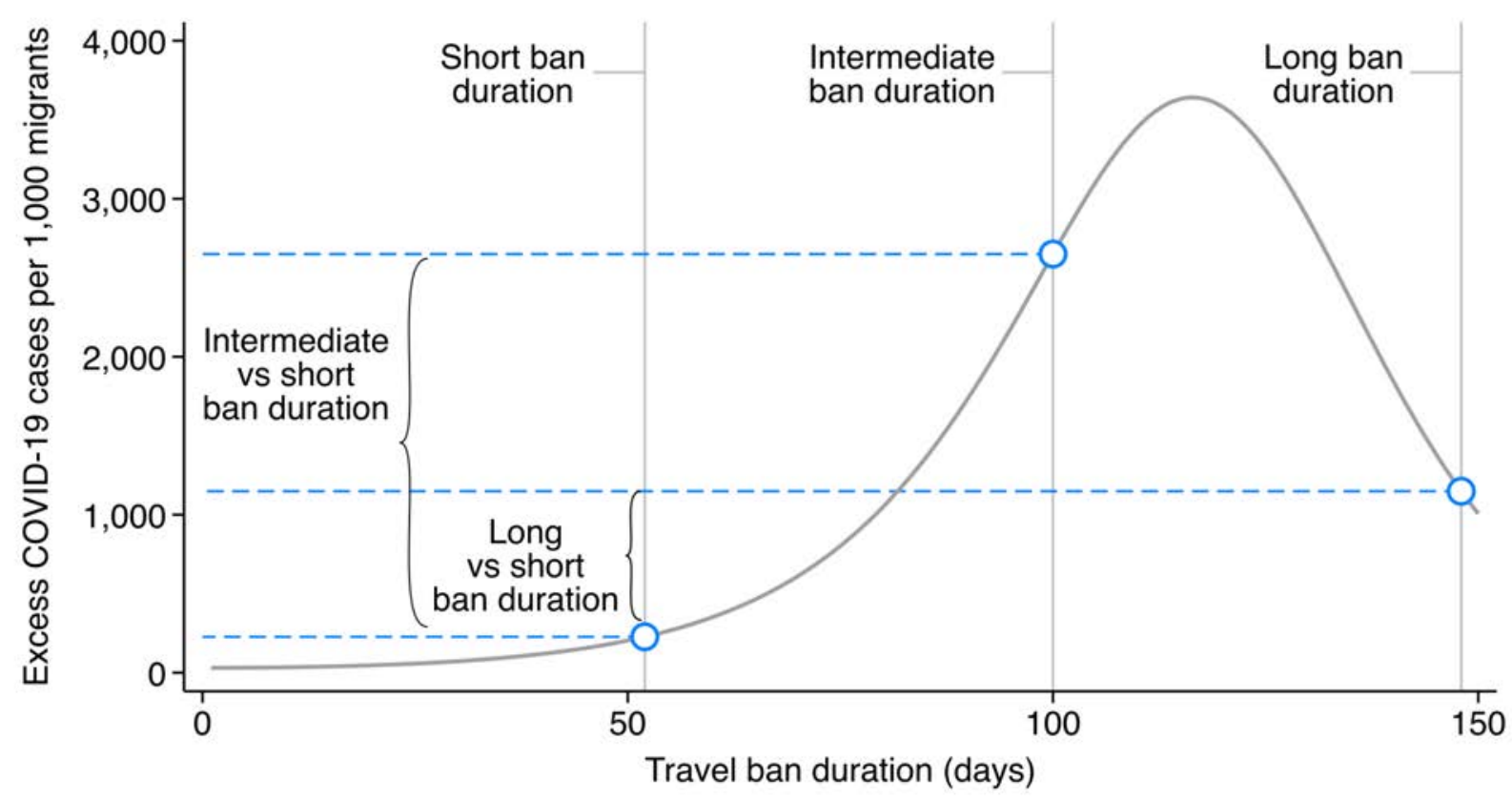

Notes: This figure plots SEIR model simulations of cumulative excess rural sink infections per 1,000 migrants (vertical axis) following the release of travel bans of varying durations (horizontal axis). For all simulations, we use a fixed end date of $t=189$ days after the ban begins. We compare excess rural sink cases to a counterfactual in which migrants leave the source immediately. Vertical gray lines mark hypothetical short, long, and intermediate bans; blue markers indicate excess cases caused by bans of these length. Brackets compare intermediate and long bans with a short ban. We calibrate this model to parameters from Mumbai, India. 
Figure 2: Results from Mumbai natural experiment
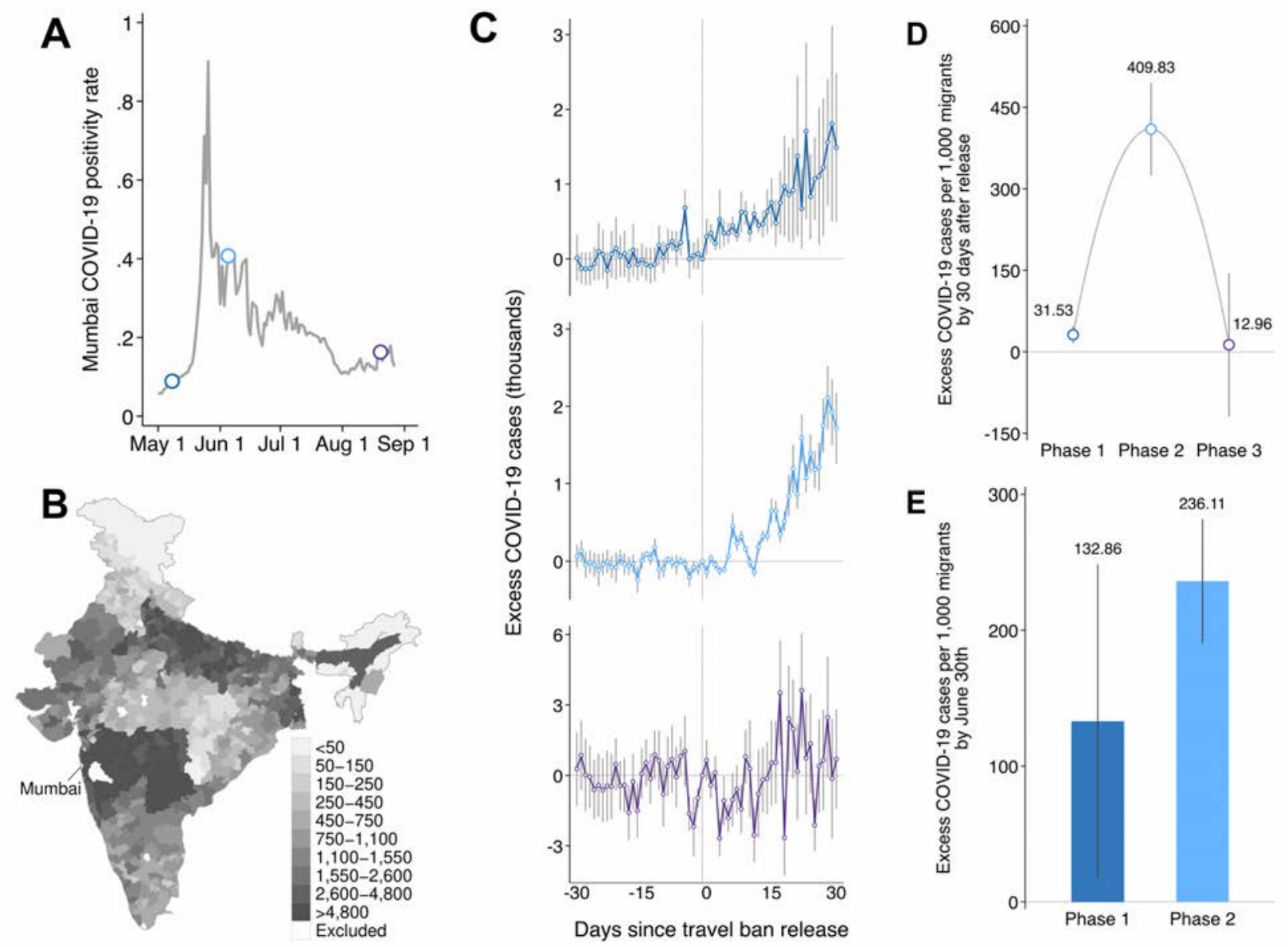

Notes: Panel A shows the Mumbai COVID-19 case positivity rate time series (moving average of prior 7 days). Dots mark the Phase 1 (dark blue), Phase 2 (light blue), and Phase 3 (purple) release dates. Panel B, shows a map of home districts of Mumbai migrants, using data from the 2011 Census of India. Panel C shows event study estimates of excess COVID-19 cases over a 30-day window before and after travel ban releases. Excess cases are shown separately for short (Phase 1, top), intermediate (Phase 2, middle), and long (Phase 3 , bottom) bans. A sharp positive trend break is visible soon after the short and intermediate ban releases. The horizontal axis shows the number of days since the travel ban release for each phase. $t=0$ is the release date. Panel D shows regression estimates of excess COVID-19 cases per 1,000 Mumbai migrants in Phase 1, Phase 2, and Phase 3 districts 30 days after travel ban releases. Panel E shows regression estimates of excess COVID-19 cases per 1,000 migrants in Phase 1 and Phase 2 districts by June 30th. Panels C-E, display $90 \%$ confidence intervals as gray vertical bars. 
Figure 3: Cross-country comparisons suggest the impact of domestic travel bans on COVID19 cases is nonlinear in their duration

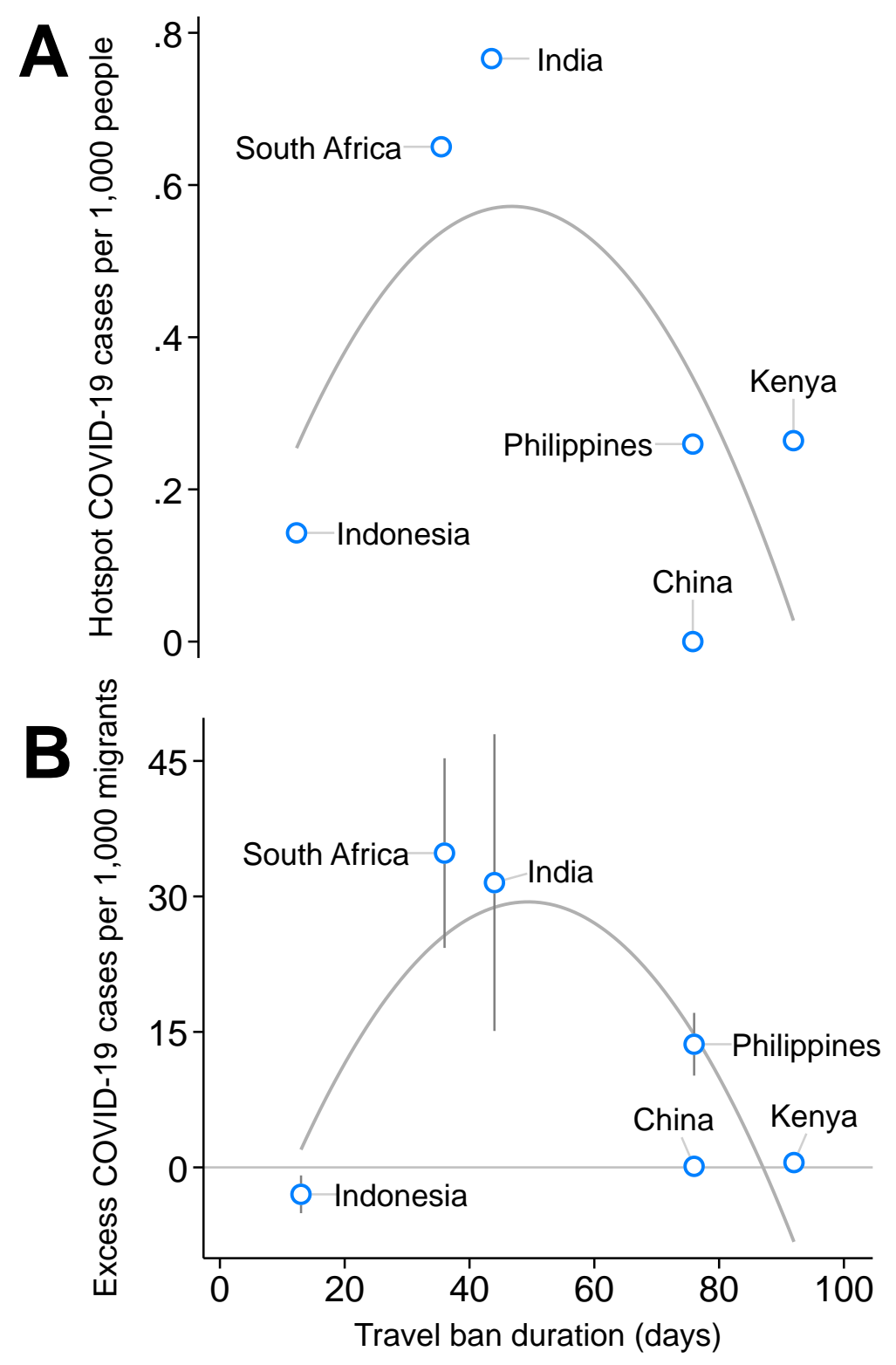

Notes: Panel A shows COVID-19 cases within one week ( \pm 7 days) of travel ban releases for urban hotspots in different countries. ${ }^{12}$ Panel B shows excess COVID-19 cases per 1,000 hotspot migrants 30 days after each country's travel ban release. 90\% confidence intervals are shown in gray vertical bars. Common horizontal axis displays ban duration. Parabolic curves in both panels are unweighted quadratic functions fit to the estimate for each country. 